\title{
PRESENTISMO Y ASPECTOS SOCIOECONÓMICOS EN MÉDICOS DE UN HOSPITAL PSIQUIÁTRICO
}

\author{
PRESENTISM AND SOCIOECONOMIC ASPECTS IN DOCTORS OF A PSYCHIATRIC HOSPITAL \\ Edgar Jesus Miraval-Rojas 1,2,a,b
}

\section{RESUMEN}

Objetivo: Determinar la relación entre el presentismo y los aspectos socioeconómicos en los médicos del Hospital Nacional Víctor Larco Herrera en el año 2018. Métodos: Se realizó un estudio cuantitativo de tipo no experimental, transversal, correlacional, prospectivo. Se trabajó con 60 médicos del Hospital Nacional Víctor Larco Herrera, la variable dependiente fue el presentismo laboral, medido con la escala presentismo laboral de Stanford SPS-6, las independientes fueron los aspectos socioeconómicos. Para el análisis bivariado, se usó la prueba de correlación de Pearson, obteniendo el coeficiente de correlación ( $r$ ) y el valor p. Resultados: $54,5 \%$ de la muestra fueron del sexo femenino, el grupo etario más frecuente fue de 28 a 37 años con 45,5\%. La prevalencia de presentismo fue de $49(81,8 \%)$ médicos. Estuvo asociada significativamente a la condición laboral $(r=-0,236$; valor $p=0,039)$, correlacionándose con el tener un régimen de contratado o por terceros frente a ser nombrado. No hubo asociación estadísticamente significativa con tener otro trabajo, personas que dependen económicamente, horario de trabajo y años de servicio. Conclusión: El presentismo en médicos del Hospital Nacional Larco Herrera estuvo asociado a la condición laboral. Se recomienda realizar más estudios en este respecto para poder conocer de mejor manera la realidad de esta problemática en nuestro País.

Palabras clave: Presentismo; Médicos; Empleo; Trabajadores. (fuente: DeCS BIREME)

\begin{abstract}
Objectives: Determine the relationship between presenteeism and socioeconomic aspects in doctors of the Hospital Nacional Víctor Larco Herrera in 2018. Methods: A non-experimental, cross-sectional, correlational, prospective quantitative study was carried out. 60 doctors from the Hospital Nacional Víctor Larco Herrera were included, the dependent variable was occupational presenteeism, measured with the Stanford SPS6 work presenteeism scale, the independent variables were the socioeconomic aspects. For the bivariate analysis, the Pearson correlation test was used, obtaining the correlation coefficient $(r)$ and the $p$ value. Results: $54.5 \%$ of the sample were female, the most frequent age group was 28 to 37 years old with $45.5 \%$. The prevalence of presenteeism was $49(81.8 \%)$. It was significantly associated to the labor condition ( $r$ $=-0.236$, value $p=0.039$ ), correlating with having a contracted or third-party regime against permanent contract worker. There was no statistically significant association with having another job, people who depend economically, work hours and years of service. Conclusion: The presenteeism in doctors of the Larco Herrera National Hospital was associated to the labor condition. It is recommended to carry out more studies in this regard in order to better understand the reality of this problem in our country.
\end{abstract}

Key words: Presenteeism; Physicians; Employment; Workers. (source: MeSH NLM)

\footnotetext{
${ }^{1}$ Hospital Victor Larco Herrera, Lima-Perú.

${ }^{2}$ Facultad de Medicina Humana, Universidad Nacional Federico Villarreal. Lima, Perú.

a Médico psiquiatra.

${ }^{\text {b}}$ Doctor en Salud Pública.
}

Citar como: Edgar Jesus Miraval-Rojas. Presentismo y aspectos socioeconómicos en médicos de un hospital psiquiátrico. Rev. Fac. Med. Hum. Octubre 2019; 19(4):22-30. DOI 10.25176/RFMH.v19i4.2340 


\section{INTRODUCCIÓN}

Recientemente el presentismo se ha convertido en un tema de interés. Adrián y col ${ }^{1}$ definieron al presentismo laboral como acudir a trabajar estando con algún problema de salud y surge como una contraparte al ausentismo laboral'. Contrario a lo que podríamos pensar intuitivamente, existen afirmaciones de que trabajar mientras se está enfermo causa una pérdida de productividad mucho mayor que el ausentismo ${ }^{2}$ y por la idea de que gestionar el presentismo de manera efectiva podría brindar una ventaja competitiva ${ }^{3}$.

Los estudios acerca del presentismo no son recientes, éste tuvo su origen en estudios europeos y estadounidenses hechos por especialistas en salud ocupacional y administración ${ }^{4}$, preocupados por la inseguridad laboral que se deriva de la reducción de personal y la reestructuración que obliga a exagerar los niveles de asistencia que resultan en estrés y enfermedad, así como el impacto de ciertas condiciones médicas generales o específicas (por ejemplo la migraña) en la productividad laboral 5 . Todo esto impulsado por el hecho de que en los últimos años, el costo creciente de la atención médica, combinado con la provisión por parte de los empleadores de planes de salud para empleados, ha llevado a evaluar el impacto económico de la salud de los trabajadores ${ }^{6,7}$.

A pesar de estos antecedentes, los estudios hechos sobre el presentismo en nuestro país son realmente escasos, y la mayoría de estos han sido realizados en otros rubros que no son los de salud ${ }^{8-10}$, por ello son pocos los profesionales de la salud que estén habituados al término. Por ser uno de los principales problemas frente a los que se tienen que oponerse las empresas, las que manejan trabajadores que se dedican a brindar un servicio de salud a población con problemas de salud mental, tienen un mayor desafío por delante. La mayoría de estudios realizados sobre presentismo laboral en personal de salud son hechos, como cabría esperar, en hospitales generales ${ }^{1,11-14}$, no habiendo estudios en hospitales dedicados especialmente a la atención en problemas de salud mental, como es el Hospital Nacional Víctor Larco Herrera de nivel III-E. Es por esto que el objetivo del presente estudio fue determinar la relación entre el presentismo y los aspectos socioeconómicos en los Médicos Del Hospital Nacional Víctor Larco Herrera en el año 2018.

\section{MÉTODOS}

Tipo y diseño de la investigación: Se realizó un estudio cuantitativo de tipo no experimental, transversal, correlacional, prospectivo.

Población y muestra: La población estuvo constituida por el total de médicos del Hospital Nacional Víctor Larco Herrera durante el año 2018, los cuales constan en 77 médicos. Se incluyeron a los todos los médicos del Hospital Nacional Víctor Larco Herrera que aceptaron desarrollar la encuesta y se excluyeron a los médicos que no estuvieron presentes al momento de la encuesta (por vacaciones, descanso médico o licencia). Después de aplicar los criterios de selección, se quedó con una muestra de 60 médicos.

Variables: La variable dependiente fue el presentismo laboral, medido con la escala presentismo laboral de Stanford SPS-6; mientras que las variables independientes fueron los aspectos socioeconómicos, siendo estos: 1)¿Cuál es su condición laboral? 2) ¿Cuántas personas dependen económicamente de Ud.? 3)¿Cuántos años de servicio tiene en el cargo? 4)¿Tiene Ud. otro trabajo? 5)¿Qué horario de trabajo tiene?, además de las características generales de la población.

Instrumento: El instrumento usado para recoger la variable dependiente fue la escala presentismo laboral de Stanford SPS-615, cuya traducción fue validada mediante el sometimiento a juicio de 3 expertos que reúnen las características de ser administradores hospitalarios y expertos (ex director del Instituto Nacional del Niño Breña, experto e investigador de la Facultad de Medicina de la Universidad Nacional Federico Villarreal y ex Director General del Instituto de Ciencia Neurológicas) y a la aplicación de la prueba piloto al 15\%, obteniéndose coeficientes de validez y confiabilidad aceptables para el estudio.

Procedimientos: Se realizaron las coordinaciones con el Cuerpo Médico del centro hospitalario a fin de lograr su aprobación, se aplicó la encuesta en los servicios donde laboran los profesionales médicos con el 100\% de aceptación de todos los que estuvieron presentes el día de la recolección, que fueron 60 médicos.

Análisis estadístico: Para el análisis descriptivo de las variables cualitativas se usaron frecuencias y porcentajes. Para el análisis bivariado, se usó la prueba de correlación de Pearson, obteniendo el coeficiente de correlación ( $r$ ) y el valor $p$, considerándose como significativo estadísticamente los que tuvieron un valor $\mathrm{p}$ menor a 0,05 . 


\section{RESULTADOS}

Características generales de la población: Participaron del estudio 60 médicos, siendo la mayoría del sexo femenino, con $54,5 \%$ y el grupo etario más frecuente fue de 28 a 37 años con 45,5\%. Asimismo, el 64\% nació en el departamento de Lima y el área del hospital en que más médicos hay es el área de hospitalización. El 49,2\% manifiesta no tener enfermedad $y$, de los que tienen alguna enfermedad, la más común es la enfermedad respiratoria. Estos valores se pueden apreciar en la tabla 1.

Tabla 1. Características generales de la población estudiada.

\section{Variables sociales}

Frecuencia

Porcentaje

\section{Edad}

28 a 37 años

27

45,5

38 a 47 años

48 a 57 años

58 a 67 años

Sexo

Masculino

Femenino

Departamento en el que nació

Departamento de Lima

Departamento diferente a Lima

Áreas del hospital

Departamento de consulta externa y salud mental comunitaria

Departamento de hospitalización

Departamento de niños y adolescentes

Departamento de adicciones

Departamento de emergencia

Departamento de apoyo al diagnóstico

Descanso médico en los últimos 3 meses

Sí

No

\section{Enfermedad crónica}

Sí

No

Enfermedad presente en el médico

No responde

Enfermedad respiratoria

10

Enfermedad neurológica

Enfermedad osteomuscular

Enfermedad gastrointestinal

Enfermedad dérmica

77,9

20,8

79,2

Fuente: Análisis de datos. 
Análisis de presentismo y los factores socioeconómicos relacionados: Del total de población estudiada, 49 (81.8\%) médicos manifiestan que asisten a trabajar estando enfermos; mientras que 11 (18.2\%) médicos no asisten a trabajar estando enfermos (figura 1).
Los factores socioeconómicos de los médicos del Hospital Nacional Victor Larco Herrera se pueden apreciar en la tabla 2.

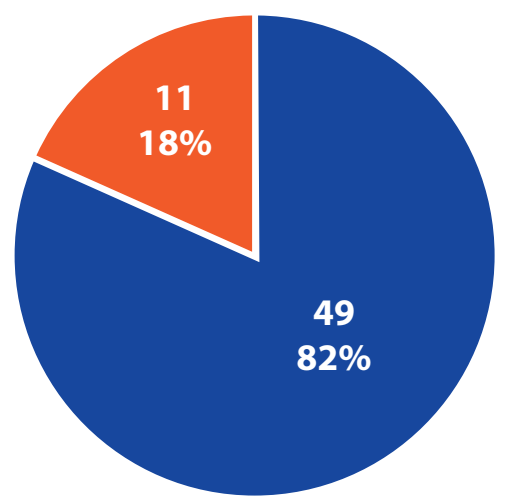

- Con presentismo laboral $\quad$ Sin presentismo laboral

Fuente: Análisis de datos.

Figura 1. Prevalencia de presentismo laboral en médicos del Hospital Nacional Victor Larco Herrera.

Tabla 2. Factores socioeconómicos de los médicos del Hospital Nacional Victor Larco Herrera.

\section{Variables socioeconómicas}

Frecuencia

Porcentaje

Otro trabajo

Sí

No

Personas que dependen económicamente del médico

Ninguno

6

22

18

14

Más de 4 personas

Condición laboral

Nombrado

50

8

2

Modalidad de tercero

Horario de trabajo

Turno fijo

40

20

10,4

Turno rotativo 
En lo que respecta a la asociación del presentismo laboral con factores socioeconómicos. Hubo una correlación negativa entre la pregunta "¿Cuál es su condición laboral?" y el presentismo laboral, obteniéndose un coeficiente de correlación de Pearson de -0,236, siendo una correlación inversa estadísticamente significativa, con un valor p de 0,039. No hubo correlaciones estadísticamente significativas entre el resto de aspectos socioeconómicos y el presentismo laboral, como se puede apreciar en la tabla 3.

Tabla 3. Correlación de los aspectos socioeconómicos con el presentismo laboral en médicos del Hospital Nacional Victor Larco Herrera.

Aspecto socioeconómico

¿Cuál es su condición laboral?

¿Cuántas personas dependen económicamente de Ud.?

¿Tiene Ud. otro trabajo?

¿Qué horario de trabajo tiene?
Coeficiente de correlación de Pearson

$-0,236$

0,175

$-0,144$

0,178

Fuente: Análisis de datos.

Causas de presentismo: Dentro de las razones por las que los trabajadores asistieron a trabajar a pesar de estar enfermos estuvieron: "Por no ausentarme del centro laboral" es la causa más frecuente de

presentismo con un porcentaje del $30 \%$, seguido de "Por no perjudicar a mis compañeros" con un $22 \%$, como se ilustra en la tabla 4.

Tabla 4. Causas de presentismo laboral en médicos del Hospital Nacional Victor Larco Herrera.

\begin{tabular}{|c|c|c|}
\hline Causa de presentismo & Frecuencia & Porcentaje \\
\hline Por no perder trabajo & 6 & $12 \%$ \\
\hline Por tener constantes descansos médicos & 2 & $4 \%$ \\
\hline Por no quedarme en casa & 4 & $8 \%$ \\
\hline Por sentirme necesaria & 4 & $8 \%$ \\
\hline Por no ausentarme del centro laboral & 14 & $33 \%$ \\
\hline Por no perjudicar a mis compañeros & 10 & $22 \%$ \\
\hline Por no perjudicar al paciente & 2 & $4 \%$ \\
\hline No se otorgó el descanso médico & 2 & $4 \%$ \\
\hline Otro & 2 & $4 \%$ \\
\hline Total & 60 & $100 \%$ \\
\hline
\end{tabular}

Fuente: Análisis de datos.

Respuestas de la escala SPS-6: En relación con la primera expresión, un importante $40 \%$ indica que estuvo en acuerdo parcialmente con que el problema de salud lefue más difícil lidiar con el estrés de su trabajo. Asimismo, en relación con la segunda expresión: “Yo me sentí sin ánimos para terminar algunas tareas de mi trabajo debido a mis problemas de salud" muestra un $40 \%$ que estuvo en acuerdo parcialmente y un $20 \%$ que estuvo en acuerdo total. En relación con la tercera expresión: "Debido a mi problema de salud no pude tener placer en mi trabajo" señala que un $37 \%$ que estuvo en acuerdo parcialmente y un $18 \%$ que estuvo en acuerdo total. En lo que respecta con la cuarta expresión:"A pesar de mi problema de salud tuve energía para terminar" el 35\% que estuvo en acuerdo parcialmente y un $37 \%$ que estuvo en acuerdo total. En la quinta expresión se observa que el $37 \%$ de los encuestados estuvo en acuerdo con que en el trabajo logro concentrarse más en sus metas a pesar de su problema de salud, mientras que el $24 \%$ estuvo en total acuerdo. Por último, el análisis de la sexta expresión, el $42 \%$ afirma con un acuerdo total que a pesar del problema de salud consiguió terminar con las tareas de su trabajo. Todo esto se observa en la tabla 5. 
Tabla 5. Evaluación de las respuestas de la escala presentismo laboral de Stanford SPS-6 por parte de los médicos del Hospital Nacional Victor Larco Herrera.

\begin{tabular}{|c|c|c|c|c|c|c|}
\hline & A & B & C & D & $E$ & $F$ \\
\hline $\begin{array}{l}\text { Debido a mi problema de salud fue más difícil } \\
\text { con el estrés en mi trabajo. }\end{array}$ & $\begin{array}{c}2 \\
(3 \%)\end{array}$ & $\begin{array}{c}7 \\
(12 \%)\end{array}$ & $\begin{array}{c}6 \\
(10 \%)\end{array}$ & $\begin{array}{c}9 \\
(15 \%)\end{array}$ & $\begin{array}{c}24 \\
(40 \%)\end{array}$ & $\begin{array}{c}12 \\
(20 \%)\end{array}$ \\
\hline $\begin{array}{l}\text { Yo me sentí sin ánimos para terminar algunas } \\
\text { tareas de mi trabajo debido a mis problemas } \\
\text { de salud. }\end{array}$ & $\begin{array}{c}2 \\
(3 \%)\end{array}$ & $\begin{array}{c}9 \\
(15 \%)\end{array}$ & $\begin{array}{c}6 \\
(10 \%)\end{array}$ & $\begin{array}{c}7 \\
(12 \%)\end{array}$ & $\begin{array}{c}24 \\
(40 \%)\end{array}$ & $\begin{array}{c}12 \\
(20 \%)\end{array}$ \\
\hline $\begin{array}{l}\text { Debido a mi problema de salud no pude tener } \\
\text { placer en mi trabajo. }\end{array}$ & $\begin{array}{c}2 \\
(3 \%)\end{array}$ & $\begin{array}{c}7 \\
(12 \%)\end{array}$ & $\begin{array}{c}7 \\
(12 \%)\end{array}$ & $\begin{array}{c}11 \\
(18 \%)\end{array}$ & $\begin{array}{c}22 \\
(37 \%)\end{array}$ & $\begin{array}{c}11 \\
(18 \%)\end{array}$ \\
\hline $\begin{array}{l}\text { A pesar de mi problema de salud tuve energía } \\
\text { para terminar mi trabajo. }\end{array}$ & $\begin{array}{c}2 \\
(3 \%)\end{array}$ & $\begin{array}{c}4 \\
(7 \%)\end{array}$ & $\begin{array}{c}4 \\
(7 \%)\end{array}$ & $\begin{array}{c}7 \\
(12 \%)\end{array}$ & $\begin{array}{c}21 \\
(35 \%)\end{array}$ & $\begin{array}{c}22 \\
(37 \%)\end{array}$ \\
\hline $\begin{array}{l}\text { En el trabajo conseguí concentrarme más en } \\
\text { mis metas a pesar de mi problema de salud. }\end{array}$ & $\begin{array}{c}2 \\
(3 \%)\end{array}$ & $\begin{array}{c}5 \\
(8 \%)\end{array}$ & $\begin{array}{c}8 \\
(13 \%)\end{array}$ & $\begin{array}{c}9 \\
(15 \%)\end{array}$ & $\begin{array}{c}22 \\
(37 \%)\end{array}$ & $\begin{array}{c}14 \\
(24 \%)\end{array}$ \\
\hline $\begin{array}{l}\text { A pesar del problema de salud conseguí } \\
\text { terminar con las tareas difíciles de mi trabajo }\end{array}$ & $\begin{array}{c}2 \\
(3 \%)\end{array}$ & $\begin{array}{c}5 \\
(8 \%)\end{array}$ & $\begin{array}{c}2 \\
(3 \%)\end{array}$ & $\begin{array}{c}4 \\
(7 \%)\end{array}$ & $\begin{array}{c}22 \\
(37 \%)\end{array}$ & $\begin{array}{c}25 \\
(42 \%)\end{array}$ \\
\hline
\end{tabular}

Fuente: Análisis de datos. Leyenda: A: No responde B: En desacuerdo totalmente C: En desacuerdo parcialmente D: Ni de acuerdo ni desacuerdo E: En acuerdo parcialmente F: En acuerdo totalmente.

\section{DISCUSIÓN}

La presente investigación es importante en nuestro medio ya que aporta datos estadísticos actuales de la problemática del presentismo en el personal médico dedicado a la atención de la salud mental, así como también datos sociodemográficos en este grupo profesional poco estudiado pero de gran importancia y responsabilidad en la salud pública. A diferencia de estudios anterior de presentismo en nuestro País, los cuales fueron centrados principalmente en médicos de guardia o que trabajan en urgencias, teniendo repercusiones como un mayor estrés en los trabajadores ${ }^{13}$, se brindan datos acerca de las implicancias y las posibles causas de este problema en un nosocomio dedicado a la salud mental, la cual tiene sus propias implicancias. Es necesario recordar que el presentismo puede tener consecuencias graves sobre la salud del trabajador, como la ansiedad y el estrés ${ }^{16,17}$; lo que hace que sea de gran importancia estudiar de mejor manera este problema en nuestro País.

En el presente estudio la muestra estuvo conformada por un $54,5 \%$ de sexo femenino, este resultado indica que hay ligeramente mayor número de mujeres que de hombres en la muestra estudiada, la diferencia de sexo en esta profesión puede ser explicada por la conformación de la población peruana y también por el incremento de la participación cada vez más activa de la mujer tanto en lo profesional como laboral.
Asimismo, el rango de edad más frecuente estuvo entre 28 y 37 años, lo cual indica que la mayoría de médicos participantes en el estudio son médicos jóvenes.

El análisis de los resultados demuestra que el $81,8 \%$ de profesionales médicos manifiestan que asisten a trabajar estando enfermos, esta prevalencia en profesionales de la salud suele ser mayor que otros profesionales, como lo reporta el estudio de Reyes Revuelta ${ }^{12}$, quien señala al grupo de enfermería como uno de los grupos profesionales con tasas significativamente más altas en comparación con otras profesiones. Sin embargo, esta prevalencia de presentismo es ligeramente diferente a la encontrada en los profesionales médicos del Servicio de Salud del Principado de Asturias, donde se encontró una prevalencia de presentismo de $52,9 \%$ del profesional médico participante en tal estudio ${ }^{13}$. Así también, fue mayo al porcentaje encontrado en 580 médicos de atención primaria de 9 hospitales en la provincia de Shaanxi, en un estudio realizado en China; la cual resultó en $47,4 \%{ }^{18}$. Asimismo, en un estudio realizado en enfermeras en el Hospital Universitario de Sao Paulo, Brasil; se encuentra una prevalencia de presentismo de $23,7 \%{ }^{19}$. Estas diferencias entre proporciones de presentismo se deben a diversos factores, en primera instancia, un poco concordancia entre las metodologías realizadas en los diversos 
estudios que abordan el tema del presentismo ${ }^{20}$, así como también pueden existir diferencias culturales en la población extranjera frente a la peruana, ya que un estudio realizado en universitarios en nuestro País encontró que los niveles de presentismo son superiores a 76,3\%8; aunque esto también pueda deberse a las características propias de un estudiante universitario, también es importante considerar que en el presente estudio y el realizado en estudiantes universitarios en el Perú tiene una población relativamente joven, en comparación con los otros estudios mencionados, lo que indicaría que un profesional joven acepta la idea de ir a trabajar estando enfermo o por motivos laborales como el miedo de ser sancionado o perder el trabajo. Lo poco claro que queda esta cuestión hace que sea necesario aumentar el número de publicaciones de los estudios sobre el presentismo en nuestro País, para poder entender estas dimensiones de mejor manera.

En lo que respecta a los factores asociados, se encontró que existió una relación estadísticamente significativa con la condición laboral, encontrándose que los que son contratados y contratados por terceros tienen un mayor índice de presentismo que los nombrados. Esto podría ser explicado por los motivos que expresaban los profesionales para asistir estando enfermo. Siendo los motivos más frecuentes los que lo hacen por no ausentarse del centro laboral y los que lo hacen por no perjudicar a los compañeros, asi como los que lo hacen por no perder el trabajo. Estas razones con mayores porcentajes nos permiten analizar que el profesional asiste a trabajar enfermo por preocupaciones laborales, el miedo de recibir sanciones o incluso ser despedido, por compañerismo o identidad institucional, coloquialmente llamado "amor a la camiseta". El estudio de Bierla et al. ${ }^{21}$ respalda al presente análisis puesto que refiere que el motivo por el cual va a trabajar estando enfermos es el miedo a perder el trabajo y por consecuencia una disminución de sus ingresos. Este análisis preocupa, pues nos indica que la salud y bienestar personal del profesional pasa a un segundo plano y no es razón suficiente para ausentarse del trabajo aun estando enfermo, la misma conclusión nos dice Petit Rodríguez et al. ${ }^{22}$ quien señala que la calidad de vida de trabajadores con presentismo está alterada en distintas áreas como el de la salud y familiar. Un dato demográfico interesante de mencionar es que la mayoría de los médicos tienen personas que dependen económicamente de ellos y eso es un motivo suficiente para laborar en más de una institución a la vez, lo cual puede relacionarse con los sueldos o la carencia de profesionales en el área.

Por otro lado, es preocupante conocer que el 20.8\% de los Médicos del Hospital Nacional Victor Larco
Herrera afirman sufrir de una enfermedad crónica, siendo las enfermedades respiratorias las más frecuente dentro de este grupo con el 6,5\%; seguidas de enfermedades gastrointestinales con el 5,2\% y enfermedades osteomusculares con el 3.9\%. Al conocer las implicancias de padecer una enfermedad crónica es conveniente señalar que en los últimos tres meses un $22,1 \%$ de los profesionales médicos refieren haber tenido descanso médico. En otro estudio se puede observar una distribución diferente de las enfermedades de los trabajadores de salud, siendo el principal las enfermedades osteomusculares $(60,9 \%)$, y en menor medida las cefaleas y migrañas $(13,9 \%)$ y las enfermedades gastrointestinales $(12,6 \%)^{19}$. Esto puede deberse a la gran participación que tiene en el Hospital donde se realizó el presente estudio el ambiente e infraestructura antigua y cerca al mar. En relación con otras investigaciones, Ruiz Gutierrez et al. ${ }^{23}$ reporta que las enfermedades que afectan al personal con presentismo son problemas osteomusculares, depresión, cefaleas, enfermedades respiratorias y también enfermedades cardiacas. Otro estudio señala que la patología más frecuente con alto índice de presentismo fue las osteomuscular ${ }^{24}$, ambas investigaciones, en resumen, respaldan nuestros hallazgos.

Al analizar las respuestas de la escala SPS-6 encontramos que un gran porcentaje de la población estudiada indica que debido a su problema de salud le fue más difícil lidiar con el estrés de su trabajo, así como también una buena proporción se sintió sin ánimos para terminar algunas tareas de su trabajo debido a sus problemas de salud. En esa misma línea, un poco más de la mitad de encuestados manifiesta que debido a su problema de salud no pudo tener placer en su trabajo. Estos análisis conllevan afirmar que el asistir estando enfermo, es decir el presentismo, ocasiona problemas en el desempeño laboral y en el bienestar individual. Por otro lado, una importante mayoría indica que a pesar de su problema de salud tuvo energía para terminar su trabajo, una proporción similar afirma que a pesar de su problema de salud consiguió terminar con las tareas de su trabajo y también, una buena proporción refiere que en el trabajo logro concentrarse más en sus metas a pesar de su problema de salud. Estas afirmaciones pueden representar los componentes que justifiquen el asistir a laborar estando enfermo y del sentido positivo que se expresa en dicha conducta. Sin embargo, no olvidemos que el presentismo encontrado en el presente estudio también está relacionado su incidencia a profesionales jóvenes y también al hecho que una buena parte de la 
población no goza de una estabilidad laboral al no ser personal nombrado.

\section{CONCLUSIÓN}

Se concluye que el presentismo en médicos del Hospital Nacional Larco Herrera estuvo asociado a la condición laboral. Se recomienda realizar más estudios en este respecto para poder conocer de mejor manera la realidad de esta problemática en nuestro País.
Contribuciones de autoría: El autor realizó la generación, redacción y aprobación final del artículo original.

\section{Financiamiento: Autofinanciado.}

Conflicto de interés: El autor trabaja en la institución hospitalaria donde se hizo el estudio. El autor no tiene otro conflicto de interés que declarar.

Recibido: 02 julio del 2019

Aprobado: 10 agosto del 2019

\section{Correspondencia: Edgar Miraval Rojas.}

Dirección: Av. Pérez Aranibar 600, Magdalena del Mar, Lima-Perú.

Teléfono: 998776732

Correo:edgarmiravalrojas@hotmail.com

\section{REFERENCIAS BIBLIOGRÁFICAS}

1. Lazo Páez A, Roldán Abellán B. Implicaciones del presentismo en la productividad laboral del área de la salud. Med Leg Costa Rica. 2015;32(1). Disponible en: https://www.scielo.sa.cr/scielo. php?script=sci_arttext\&pid=S1409-00152015000100018

2. Collins JJ, Baase CM, Sharda CE, Ozminkowski RJ, Nicholson $S$ Billotti GM, et al. The assessment of chronic health conditions on work performance, absence, and total economic impact for employers. J Occup Environ Med. junio de 2005;47(6):547-57. Disponible en: 10.1097/01.jom.0000166864.58664.29

3. Hemp P. Presenteeism: At Work-But Out of It. Harvard Business Review [Internet]. 2004 [citado 16 de junio de 2019];(October 2004). Disponible en: https://hbr.org/2004/10/presenteeism-at-work-but-out-of-it

4. Johns G. Presenteeism in the workplace: A review and research agenda. J Organ Behav. 2010;31(4):519-42. Disponible en: https://doi. org/10.1002/job.630

5. Koopmanschap M, Burdorf A, Jacob K, Meerding WJ, Brouwer W, Severens $\mathrm{H}$. Measuring productivity changes in economic evaluation setting the research agenda. PharmacoEconomics. 2005;23(1):47-54 Disponible en: https://doi.org/10.2165/00019053-200523010-00004

6. Guest JF, Ayoub N, Mcllwraith T, Uchegbu I, Gerrish A, Weidlich D, et al. Health economic burden that different wound types impose on the UK's National Health Service. Int Wound J. 2017;14(2):322-30. Disponible en: 10.1111/iwj.12603

7. Listl S, Galloway J, Mossey PA, Marcenes W. Global Economic Impact of Dental Diseases. J Dent Res. 1 de octubre de 2015;94(10):1355-61. Disponible en: https://doi.org/10.1177/0022034515602879

8. Chafloque-Céspedes R, Vara-Horna A, Lopez-Odar D, Santi-Huaranca I, Diaz-Rosillo A, Asencios-Gonzalez Z. Ausentismo, presentismo y rendimiento académico en estudiantes de universidades peruanas. Propósitos Represent. enero de 2018;6(1):83-133. Disponible en: http:// dx.doi.org/10.20511/pyr2018.v6n1.177

9. Arteaga-Cubas G. Impacto de la violencia contra las mujeres en el presentismo y productividad de una empresa bancaria y su relación con los ataques de la clientela. Lima - Perú: 2014. San Martín Emprend. 2016;7(2):3-26. Disponible en: https://www.sme.usmp.edu.pe/index. $\mathrm{php} / \mathrm{sme} / \mathrm{article} / \mathrm{view} / 81$

10. García M de A. Problemática multifactorial del absentismo laboral, el presentismo y la procrastinación en las estructuras en que se desenvuelve el trabajador. Med Segur Trab. junio de 2011;57(223):11120. Disponible en: http://scielo.isciii.es/scielo.php?script=sci arttext\&pid=S0465-546X2011000200004\&lng=es\&nrm=iso\&tlng=es

11. Vera-Calzaretta A, Carrasco-Dájer C, Costa S da, Páez-Rovira D. Factores psicosociales del presentismo en trabajadores del Sistema de
Salud chileno. Rev Psicol Trab Las Organ. agosto de 2015;31(2):119-28. Disponible en: http://dx.doi.org/10.1016/j.rpto.2015.03.004

12. Reyes Revuelta JF. Presentismo en Enfermería. Implicaciones en seguridad del paciente: posibilidades de control y reducción. Enferm Glob. julio de 2014;13(35):362-73. Disponible en: http://scielo.isciii.es/ scielo.php?script=sci_arttext\&pid=S1695-61412014000300020

13. Sanchez-Zaballos M, Baldonedo-Mosteiro M, Mosteiro-Diaz MP. Riesgos psicosociales en profesionales sanitarios de los servicios de urgencias: presentismo. EMERGENCIAS [Internet]. 22 de diciembre de 2017 [citado 16 de junio de 2019];30(1). Disponible en: http:// emergenciasojs.gruposaned.com/index.php/emergencias/article/ view/705

14. Rojas R. Enfoque del presentismo en empresas de salud. Cienc Trab. 2007;9(24):64-8. Disponible en: http://bases.bireme.br/cgi-bin/wxislind. exe/iah/online/?IsisScript=iah/iah

15. López Roza EM, Riaño Casallas MI. Presentismo y su relación con la seguridad y salud en el trabajo: una revisión de la literatura. Mov Científico. 2015;9(1):50-9. Disponible en: https://revistas.ibero.edu.co/ index.php/Rmcientifico/article/view/856

16. Yang T, Ma M, Zhu M, Liu Y, Chen Q, Zhang S, et al. Challenge or hindrance: Does job stress affect presenteeism among Chinese healthcare workers? J Occup Health. 19 de diciembre de 2017;17-0195OA. Disponible en: https://doi.org/10.1539/joh.17-0195-OA

17. Laing SS, Jones SMW. Anxiety and Depression Mediate the Relationship Between Perceived Workplace Health Support and Presenteeism: A Cross-sectional Analysis. J Occup Environ Med. noviembre de 2016;58(11):1144. Disponible en: https://doi.org/10.1097/ JOM.0000000000000880

18. Tang $\mathrm{N}$, Han L, Yang $\mathrm{P}$, Zhao $\mathrm{Y}$, Zhang $\mathrm{H}$. Are mindfulness and selfefficacy related to presenteeism among primary medical staff: A crosssectional study. Int J Nurs Sci. 10 de abril de 2019;6(2):182-6. Disponible en: https://doi.org/10.1016/j.ijnss.2019.03.004

19. Silva FJ, Felli VE, Martinez MC, Silva SM, Baptista PC, Borges EM, et al. P142 Presenteeism in brazilian care nursing workers. Occup Environ Med. 1 de septiembre de 2016;73(Suppl 1):A168-A168. Disponible en: http://dx.doi.org/10.1136/oemed-2016-103951.459

20. Lui JNM, Andres EB, Johnston JM. Presenteeism exposures and outcomes amongst hospital doctors and nurses: a systematic review. BMC Health Serv Res. 19 de diciembre de 2018;18(1):985. Disponible en: 10.1186/s12913-018-3789-z

21. Bierla I, Huver B, Richard S. Presenteeism at work: The influence of managers. Int J Bus Manag Stud. 1 de diciembre de 2011;3(2):97-107. Disponible en: https://dergipark.org.tr/download/article-file/255947 
22. Petit Rodríguez C, Sánchez López C, Vargas Valenzuela N. El presentismo: un estudio descriptivo de la alteración de la calidad de vida de un trabajador de pequeñas y mediandas empresas [Internet] [Tesis de licenciatura]. [Santiago, Chile.]: Universidad Academia de Humanismo Cristiano; 2012 [citado 17 de junio de 2019]. Disponible en: http://bibliotecadigital.academia.cl/handle/123456789/2603

23. Ruiz Gutiérrez F, Palomino Baldeón J, Zambrano Beltrán R, Llap Yesán C. Prevalencia, impacto en la productividad y costos totales de las principales enfermedades en los trabajadores de un hospital al sur del Perú en el año 2003.: Prevalence and economy impact. Rev Medica Hered. enero de 2006;17(1):28-34. Disponible en: http://www.scielo. org.pe/scielo.php?pid=s1018-130x2006000100006\&script=sci_arttext

24. España G. P. Percepción del presentismo y comportamiento de la morbilidad en trabajadores de una industria papelera, Carabobo 2010-2011 [Tesis de especialidad]. [Carabobo, Venezuela.]: Universidad de Carabobo; 2013. Disponible en: http://mriuc.bc.uc.edu.ve/ handle/123456789/1117

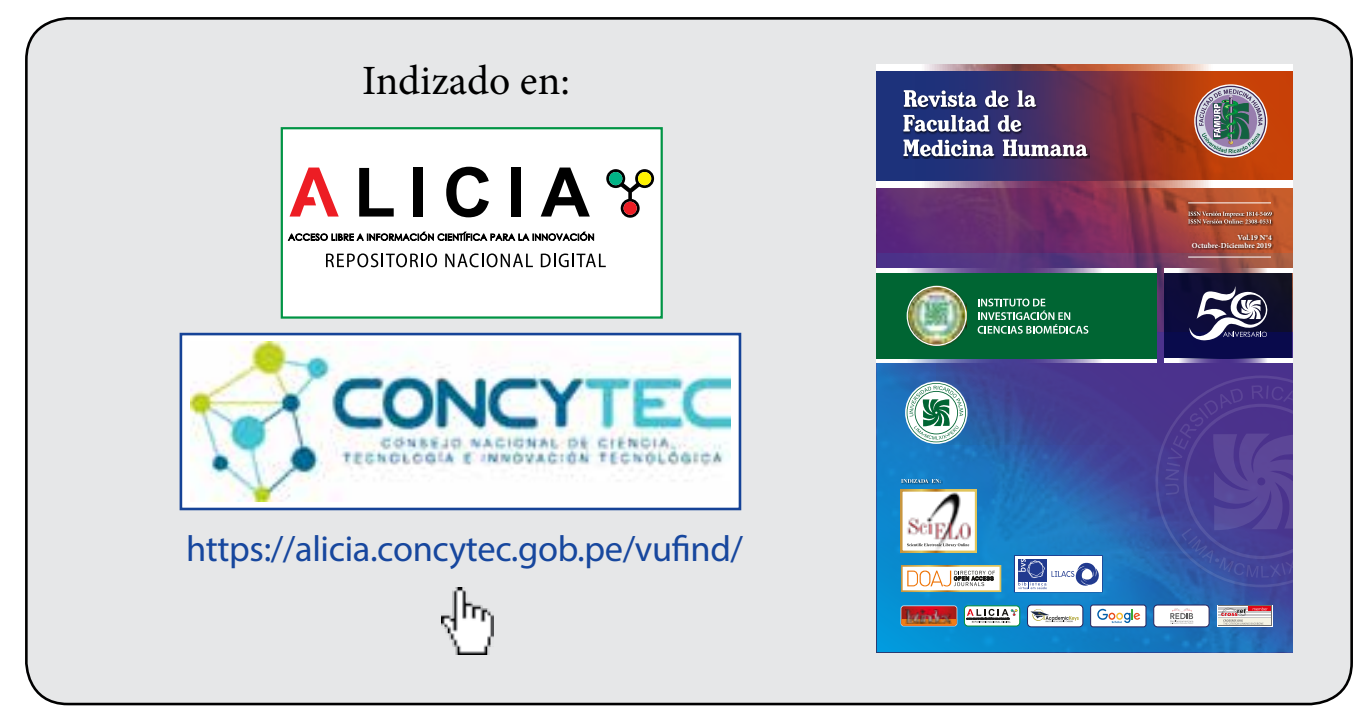

